\title{
Ancillary Ligand Effects on C-H Bond Activation Reactions Promoted by $\beta$-Diiminate Iridium Complexes.
}

\author{
Wesley H. Bernskoetter, Emil Lobkovsky, and Paul J. Chirik* \\ Department of Chemistry and Chemical Biology, Baker Laboratory \\ Cornell University, Ithaca NY 14853. \\ -- Supporting Information --
}




\section{Table of Contents}

General Considerations and Additional Experimental Procedures $\quad$ S3

$\begin{array}{ll}\text { Crystallographic Data for (BDI-3) } \operatorname{Ir}(\mathrm{COD}) & \mathrm{S} 12\end{array}$

$\begin{array}{ll}\text { Crystallographic Data for (BDI-5) } \operatorname{Ir}(\mathrm{COD}) & \text { S16 }\end{array}$

$\begin{array}{ll}\text { Crystallographic Data for } 2 & \text { S21 }\end{array}$

$\begin{array}{ll}\text { References } & \text { S25 }\end{array}$ 


\section{Additional Experimental Procedures}

General Considerations. All air- and moisture-sensitive manipulations were carried out using standard high vacuum line, Schlenk or cannula techniques or in an M. Braun inert atmosphere drybox containing an atmosphere of purified nitrogen. The M. Braun drybox was equipped with a cold well designed for freezing samples in liquid nitrogen. Solvents for air- and moisture-sensitive manipulations were dried and deoxygenated using literature procedures. ${ }^{1}$ Deuterated solvents for NMR spectroscopy were purchased from Cambridge Isotope Laboratories and were distilled from sodium metal under an atmosphere of argon and stored over $4 \AA$ molecular sieves. Hydrogen and argon gas were purchased from Airgas Incorporated and passed through a column containing manganese oxide supported on vermiculite and $4 \AA$ molecular sieves before admission to the high vacuum line. The following compounds were prepared according to literature procedures: $\left({ }^{\mathrm{iPr}} \mathrm{BDI}\right) \operatorname{Ir}(\mathrm{COE}) \mathrm{N}_{2},{ }^{2}\left[\mathrm{Li}\left(\mathrm{OEt} \quad{ }_{2}\right)\right][\mathrm{BDI}-1],\left[\mathrm{Li}\left(\mathrm{OEt} \quad{ }_{2}\right)\right][\mathrm{BDI}-2],\left[\mathrm{Li}\left(\mathrm{OEt} \quad{ }_{2}\right)\right][\mathrm{BDI}-3],{ }^{3}$ $\left[\mathrm{Li}\left(\mathrm{OEt}_{2}\right)\right][\mathrm{BDI}-4],{ }^{4}\left[\mathrm{Li}\left(\mathrm{OEt}_{2}\right)\right][\mathrm{BDI}-5],{ }^{5}\left[\mathrm{Ir}(\mathrm{COE})_{2} \mathrm{Cl}\right]_{2}$ and $[\operatorname{Ir}(\mathrm{COD}) \mathrm{Cl}]_{2}{ }^{6}$

${ }^{1} \mathrm{H}$ spectra were recorded on Varian Mercury 300, Inova 400 and 500 spectrometers operating at 299.763, 399.780 and $500.62 \mathrm{MHz}$, respectively. ${ }^{13} \mathrm{C} \mathrm{NMR}$ spectra were obtained on Varian Mercury 300 and Inova 500 spectrometers operating at 75.37 and $100.52 \mathrm{MHz}$, respectively. All chemical shifts are reported relative to $\mathrm{SiMe}_{4}$ using ${ }^{1} \mathrm{H}$ (residual) chemical shifts of the solvent as a secondary standard. ${ }^{2} \mathrm{H}$ NMR spectra were recorded on a Varian Inova 500 spectrometer operating at $76.851 \mathrm{MHz}$ and the spectra were referenced using an internal benzene- $d_{6}$ standard. ${ }^{31} \mathrm{P}$ NMR spectroscopy was carried out on a Varian Inova 400 spectrometer operating at $161.83 \mathrm{MHz}$ and spectra were referenced to an external $\mathrm{H}_{3} \mathrm{PO}_{4}$ standard. ${ }^{19} \mathrm{~F}$ NMR spectroscopy was conducted on 
a Varian Inova 400 spectrometer operating at 376.127 MHz. Spectra were referenced to an external hexaflourobenzene standard. Infrared spectroscopy was conducted on a Mattson RS-10500 Research Series FT-IR spectrometer calibrated with a polystyrene standard.

Single crystals suitable for X-ray diffraction were coated with polyisobutylene oil in a drybox and were quickly transferred to the goniometer head of a Siemens SMART CCD Area detector system or a Bruker X8 APEX2 system equipped with a molybdenum X-ray tube $(\lambda=0.71073$ A). Preliminary data revealed the crystal system. A hemisphere routine was used for data collection and determination of lattice constants. The space group was identified and the data were processed using the Bruker SAINT program and corrected for absorption using SADABS. The structures were solved using direct methods (SHELXS) completed by subsequent Fourier synthesis and refined by fullmatrix least-squares procedures. Elemental analyses were performed at Robertson Microlit Laboratories, Inc., in Madison, NJ.

Preparation of (BDI-2) $\operatorname{Ir}(\mathbf{C O E}) \mathbf{N}_{2}$. This molecule was prepared in a similar manner to (BDI-1) $\operatorname{Ir}(\mathrm{COE}) \mathrm{N}_{2}$ with $0.050 \mathrm{~g}(0.056 \mathrm{mmol})$ of $\left[\mathrm{Ir}(\mathrm{COE})_{2} \mathrm{Cl}_{2}\right.$ and $0.049 \mathrm{~g}(0.111 \mathrm{mmol})$ of $\mathrm{Li}[\mathrm{BDI}-2] \cdot \mathrm{Et}_{2} \mathrm{O}$ yielding $0.028 \mathrm{~g}(48 \%)$ of brown crystals identified as (BDI2) $\operatorname{Ir}(\mathrm{COE}) \mathrm{N}_{2}$. Anal. Calcd. for $\mathrm{C}_{33} \mathrm{H}_{47} \mathrm{IrN}_{4}$ : C, 57.28; H, 6.85; N, 8.10. Found: C, 57.62; $\mathrm{H}, 6.91 ; \mathrm{N}, 7.75 .{ }^{1} \mathrm{H}$ NMR (benzene- $\left.d_{6}\right): \delta=1.00-1.22\left(\mathrm{~m}, 8 \mathrm{H}, \mathrm{CH}_{2} \mathrm{COE}\right), 1.28(\mathrm{t}, 6 \mathrm{H}, 8$ $\mathrm{Hz}, \mathrm{CH}_{2} \mathrm{Me}$ ), 1.48 (t, 6H, $8 \mathrm{~Hz}, \mathrm{CH}_{2} \mathrm{Me}$ ), 1.49 (s, 3H, $\mathrm{CH}_{3}$ ), 1.72 (s, 3H, $\mathrm{CH}_{3}$ ), 2.23 (br s, 2H, $\mathrm{CH}_{2} \mathrm{COE}$ ), 2.42 (m, 2H, $\mathrm{CH}_{2} \mathrm{Me}$ ), 2.67 (br d, 2H, $\mathrm{CH} \mathrm{COE}$ ), 2.79 (m, 2H, $\mathrm{CH}_{2} \mathrm{Me}$ ), $2.93\left(\mathrm{~m}, 2 \mathrm{H}, \mathrm{CH}_{2} \mathrm{Me}\right), 3.17\left(\mathrm{~m}, 2 \mathrm{H}, \mathrm{CH}_{2} \mathrm{Me}\right), 5.34(\mathrm{~s}, 1 \mathrm{H}, \mathrm{CH}), 7.19-7.26(\mathrm{ArH}) .{ }^{13} \mathrm{C}$ 
NMR (benzene- $\left.d_{6}\right): \delta=13.69,14.35,31.34\left(\mathrm{CH}_{2} \mathrm{COE}\right), 25.07,25.92,\left(\mathrm{CH}_{2} \mathrm{Me}\right), 24.00$, $24.10(\mathrm{CMe}), 26.75,28.23\left(\mathrm{CH}_{2} \mathrm{Me}\right), 59.16(-\mathrm{CH} \mathrm{COE}), 101.30(\mathrm{CH}), 125.58,126.31(p-$ Ar), 126.57, $126.64(m-\mathrm{Ar}), 137.41,138.30(o-\mathrm{Ar})$. IR (benzene- $\left.d_{6}\right): v_{\mathrm{N}-\mathrm{N}}=2121 \mathrm{~cm}^{-1}$.

Preparation of (BDI-1)Ir(COD). A $20 \mathrm{~mL}$ scintillation vial was charged with $0.093 \mathrm{~g}$ $(0.14 \mathrm{mmol})$ of $[\operatorname{Ir}(\mathrm{COD}) \mathrm{Cl}]_{2}$ in approximately $5 \mathrm{~mL}$ diethyl ether. The resulting orange slurry was chilled in a drybox freezer to $-35^{\circ} \mathrm{C}$ for about 20 minutes after which time $0.105 \mathrm{~g}(0.280 \mathrm{mmol}) \mathrm{Li}[\mathrm{BDI}-1] \cdot \mathrm{Et}_{2} \mathrm{O}$ in approximately $5 \mathrm{~mL}$ diethyl ether was added. The orange mixture was stirred for $16 \mathrm{hrs}$ at ambient temperature. Filtration through Celite followed by solvent removal in vacuo and subsequent recrystallization from pentane at $-35{ }^{\circ} \mathrm{C}$ affords $0.053 \mathrm{~g}$ (37\%) of a yellow solid identified as (BDI-1) $\operatorname{Ir}(\mathrm{COD})$. Anal. Calcd. for $\mathrm{C}_{29} \mathrm{H}_{37} \mathrm{IrN}_{2}$ : C, 57.49; H, 6.16; N, 4.62. Found: C, 57.40; H, 5.84; N, 4.36. ${ }^{1} \mathrm{H}$ NMR (benzene- $d_{6}$ ): $\delta=1.42$ (br d, $4 \mathrm{H}, \mathrm{COD}$ ), 1.56 (s, $6 \mathrm{H}, \mathrm{CH}_{3}$ ), 2.08 (br s, $4 \mathrm{H}$, COD), $2.28(\mathrm{~s}, 12 \mathrm{H}, \mathrm{ArMe}) 3.04(\mathrm{br} \mathrm{s}, 4 \mathrm{H}, \mathrm{COD}), 5.39(\mathrm{~s}, 1 \mathrm{H}, \mathrm{CH}), 6.95-7.04(\mathrm{Ar} H) .{ }^{13} \mathrm{C}$ NMR (benzene- $\left.d_{8}\right): \delta=19.28(\mathrm{ArMe}), 25.77(\mathrm{CMe}), 31.89,63.22(\mathrm{COD}), 102.21(\mathrm{CH})$, 126.07 ( $p$-Ar), 129.00 (m-Ar), 132.97 (o-Ar).

Preparation of (BDI-3) $\operatorname{Ir}(\mathbf{C O D})$. This molecule was prepared in a similar manner to (BDI-1) $\operatorname{Ir}(\mathrm{COD})$ with $0.101 \mathrm{~g}(0.150 \mathrm{mmol})$ of $[\operatorname{Ir}(\mathrm{COD}) \mathrm{Cl}]_{2}$ and $0.151 \mathrm{~g}(0.30 \mathrm{mmol})$ $\mathrm{Li}[\mathrm{BDI}-3] \cdot \mathrm{Et}_{2} \mathrm{O}$ yielding $0.070 \mathrm{~g}(65 \%)$ of orange solid identified as $(\mathrm{BDI}-3) \operatorname{Ir}(\mathrm{COD})$. Single crystals suitable for X-ray diffraction were grown under similar conditions. Anal. Calcd. for $\mathrm{C}_{37} \mathrm{H}_{53} \mathrm{IrN}_{2}$ : C, 61.89; H, 7.44. Found: C, 61.67; H, 7.24. ${ }^{1} \mathrm{H}$ NMR (benzene$\left.d_{6}\right): \delta=1.07\left(\mathrm{~d}, 12 \mathrm{H}, 6 \mathrm{~Hz}, \mathrm{CHMe} e_{2}\right), 1.41(\mathrm{~d}, 12 \mathrm{H}, 6 \mathrm{~Hz}, \mathrm{CHMe}), 1.71\left(\mathrm{~s}, 6 \mathrm{H}, \mathrm{CH}_{3}\right), 2.06$ 
(br s, 4H, COD), 3.18 (br s, 4H, COD), 3.50 (sept, 4H, 6Hz, CHMe ${ }_{2}$ ), 5.33 (s, 1H, CH), 7.09-7.18 (ArH). ${ }^{13} \mathrm{C}$ NMR (benzene- $\left.d_{8}\right): \delta=24.46,25.04\left(\mathrm{CHMe} e_{2}\right), 26.56\left(\mathrm{CHMe}_{2}\right)$, $28.11(\mathrm{CMe}), 30.94,62.36$ (COD), $100.32(\mathrm{CH}), 123.90$ (p-Ar), 126.49 (m-Ar), 142.56 (o-Ar).

Preparation of (BDI-4)Ir(COD). This molecule was prepared in a similar manner to (BDI-1) $\operatorname{Ir}(\mathrm{COD})$ with $0.100 \mathrm{~g}(0.149 \mathrm{mmol})$ of $[\operatorname{Ir}(\mathrm{COD}) \mathrm{Cl}]_{2}$ and $0.181 \mathrm{~g}(0.298 \mathrm{mmol})$ of $\mathrm{Li}[\mathrm{BDI}-4] \cdot \mathrm{Et}_{2} \mathrm{O}$ yielding $0.027 \mathrm{~g}(11 \%)$ of red crystals identified as (BDI-4) $\operatorname{Ir}(\mathrm{COD})$. Anal. Calcd. for $\mathrm{C}_{37} \mathrm{H}_{45} \mathrm{IrN}_{2} \mathrm{~F}_{6}$ : C, 53.93; H, 5.50; N, 3.40. Found: C, 53.73; H, 5.78; N, 3.14. ${ }^{1} \mathrm{H}$ NMR (benzene- $d_{6}$ ): $\delta=1.21\left(\mathrm{~d}, 12 \mathrm{H}, 7 \mathrm{~Hz}, \mathrm{CH} M e_{2}\right.$ ), 1.41 (br s, 4H, COD), 1.44 (d, 12H, 7Hz, CHMe $^{2}, 1.99$ (br s, 4H, COD), 3.33 (sept, 4H, 7Hz, CHMe ${ }_{2}$ ), 3.38 (br s, 4H, COD), $6.52(\mathrm{~s}, 1 \mathrm{H}, \mathrm{CH}), 7.17(\mathrm{br}, 4 \mathrm{H}, m-\mathrm{Ar} H), 7.24(\mathrm{br}, 2 \mathrm{H}, p-\mathrm{Ar} H) .{ }^{13} \mathrm{C} \mathrm{NMR}$ (benzene- $\left.d_{8}\right): \delta=24.30,24.90\left(\mathrm{CHMe} e_{2}\right), 28.37\left(\mathrm{CHMe}_{2}\right), 30.44$ (COD), 67.59 (COD), $94.45(\mathrm{CH}), 123.85$ (p-Ar), 128.11 (m-Ar), $141.80(o-\mathrm{Ar}), 150.74\left(C \mathrm{~F}_{3}\right) .{ }^{19} \mathrm{~F} \mathrm{NMR}$ (benzene- $\left.d_{6}\right): \delta=108.10\left(\mathrm{~s}, \mathrm{C} F_{3}\right)$.

Preparation of (BDI-5)Ir(COD). A $20 \mathrm{~mL}$ scintillation vial was charged with $0.044 \mathrm{~g}$ $(0.066 \mathrm{mmol})$ of $[\mathrm{Ir}(\mathrm{COD}) \mathrm{Cl}]_{2}$ and $0.075 \mathrm{~g}(0.132 \mathrm{mmol})$ of $\mathrm{Li}[\mathrm{BDI}-5] \cdot \mathrm{Et}_{2} \mathrm{O}$ in approximately $15 \mathrm{~mL}$ of diethyl ether. The yellow solution was stirred at ambient temperature for 2 days and the solvent removed in vacuo leaving a yellow solid. Extraction into pentane and filtration through a pad of Celite followed by solvent removal produced a yellow powder. Recrystallization from pentane or methylene chloride at -35 ${ }^{\circ} \mathrm{C}$ afforded 0.020g (19\%) of a yellow crystalline solid identified as (BDI-5) $\operatorname{Ir}(\mathrm{COD})$. Anal. Calcd. for $\mathrm{C}_{43} \mathrm{H}_{63} \mathrm{IrN}_{2}$ : C, 64.54; H, 7.94; N, 3.50. Found: C, 64.22; H, 7.65; N, 
3.23. ${ }^{1} \mathrm{H}$ NMR (benzene- $\left.d_{6}\right): \delta=0.92\left(\mathrm{~s}, 9 \mathrm{H}, \mathrm{C}\left(\mathrm{CH}_{3}\right)_{3}\right), 1.08\left(\mathrm{~d}, 6 \mathrm{H}, 7 \mathrm{~Hz}, \mathrm{CHMe} e_{2}\right), 1.15$ (d, 6H, 7Hz, CHMe $), 1.38$ (d, 6H, 7Hz, CHMe $\left.e_{2}\right), 1.41$ (d, 6H, 7Hz, CHMe $\left.e_{2}\right), 1.77$ (br d, 4H, COD), 1.79 (s, 9H, C(CH $)_{3}$ ), 2.00 (br s, 4H, COD), 2.93 (sept, 2H, 7Hz, $\mathrm{CHMe}_{2}$ ), 3.23 (sept, 2H, 7Hz, CHMe $)$ ), 3.91 (br s, 4H, COD), 4.64 (t, 1H, 6Hz, $\eta^{6}-p-\mathrm{Ar}$ ), 5.03 (d, $\left.2 \mathrm{H}, 6 \mathrm{~Hz}, \eta^{6}-m-\mathrm{Ar}\right), 5.39(\mathrm{~s}, 1 \mathrm{H}, \mathrm{CH}), 7.15(\mathrm{br}, 4 \mathrm{H}, m-\mathrm{Ar} H), 7.30(\mathrm{br} \mathrm{s}, 2 \mathrm{H}, p-\mathrm{Ar} H) .{ }^{13} \mathrm{C}$ NMR (benzene- $\left.d_{8}\right): \delta=23.00,23.32,23.85,25.19\left(\mathrm{CHMe} e_{2}\right), 27.62,28.74\left(\mathrm{CHMe}_{2}\right)$, 30.31, $31.41\left(\mathrm{C}\left(\mathrm{CH}_{3}\right)_{3}\right), 33.73,54.85(\mathrm{COD}), 79.47\left(\eta^{6}-p-\mathrm{Ar}\right), 93.04\left(\eta^{6}-m-\mathrm{Ar}\right), 98.58$ (CH), 122.44 (p-Ar), 123.20 (m-Ar), 136.23 (o-Ar).

Preparation of (BDI-1) Ir(CO) $)_{2}$. A J. Young NMR tube was charged with $8 \mathrm{mg}(0.01$ mmol) of (BDI-1) $\operatorname{Ir}(\mathrm{COE}) \mathrm{N}_{2}$ and approximately $0.5 \mathrm{~mL}$ of benzene- $d_{6}$. On the vacuum line, the tube was submerged in liquid nitrogen, evacuated and $1 \mathrm{~atm}$ of $\mathrm{CO}$ admitted. The solution was quickly thawed and left to stand at ambient temperature for 24 hours.. The solvent and resulting cyclooctene was removed in vacuo to yielding a yellow foam. ${ }^{1} \mathrm{H}$ NMR (benzene- $\left.d_{6}\right): \delta=1.45(\mathrm{~s}, 12 \mathrm{H}, \operatorname{ArMe}), 2.03\left(\mathrm{~s}, 6 \mathrm{H}, \mathrm{CH}_{3}\right), 5.14(\mathrm{~s}, 1 \mathrm{H}, \mathrm{CH})$, 6.91-7.01 $(\mathrm{Ar} H) .{ }^{13} \mathrm{C}$ NMR (benzene- $\left.d_{6}\right): \delta=19.37(\mathrm{ArMe}), 22.32\left(\mathrm{CH}_{3}\right), 101.81(\mathrm{CH})$, 126.66 (p-Ar), $128.71(m-A r), 131.59$ (o-Ar). IR (pentane): $v_{\mathrm{CO}}=2054 \mathrm{~cm}^{-1}, 1986 \mathrm{~cm}^{-1}$.

Preparation of $(\mathbf{B D I}-2) \operatorname{Ir}(\mathbf{C O})_{2}$. This molecule was prepared in a similar manner to (BDI-1) $\operatorname{Ir}(\mathrm{CO})_{2}$ with $10 \mathrm{mg}(0.020 \mathrm{mmol})$ of $(\mathrm{BDI}-2) \operatorname{Ir}(\mathrm{COE}) \mathrm{N}_{2} .{ }^{1} \mathrm{H}$ NMR (benzene- $\left.d_{6}\right)$ : $\delta=1.25\left(\mathrm{t}, 12 \mathrm{H}, 8 \mathrm{~Hz}, \mathrm{CH}_{2} \mathrm{Me}\right), 1.53\left(\mathrm{~s}, 6 \mathrm{H}, \mathrm{CH}_{3}\right), 2.55\left(\mathrm{~m}, 4 \mathrm{H}, \mathrm{CH}_{2} \mathrm{Me}\right), 2.69(\mathrm{~m}, 4 \mathrm{H}$, $\left.\mathrm{CH}_{2} \mathrm{Me}\right), 5.18(\mathrm{~s}, 1 \mathrm{H}, \mathrm{CH}), 7.07-7.18(\mathrm{ArH}) .{ }^{13} \mathrm{C}$ NMR (benzene- $\left.d_{6}\right): \delta=14.24\left(\mathrm{CH}_{2} \mathrm{Me}\right)$, 
$22.99\left(\mathrm{CH}_{3}\right), 24.98\left(\mathrm{CH}_{2} \mathrm{Me}\right), 101.43(\mathrm{CH}), 126.18$ (p-Ar), 127.07 (m-Ar), 136.63 (o-Ar). IR (pentane): $v_{\mathrm{CO}}=2054 \mathrm{~cm}^{-1}, 1985 \mathrm{~cm}^{-1}$.

Preparation of (BDI-3) $\mathbf{I r}(\mathbf{C O})_{2}$. A thick walled glass vessel was charged with $0.012 \mathrm{~g}$ $(0.020 \mathrm{mmol})$ of $(\mathrm{BDI}-3) \mathrm{IrH}_{4}$ and approximately $15 \mathrm{~mL}$ of pentane. On the vacuum line, the vessel was submerged in liquid nitrogen, evacuated and one atmosphere of $\mathrm{CO}$ added. The contents of the vessel were thawed and the resulting solution stirred for 24 hours at ambient temperature. The pentane was removed in vacuo and the resulting yellow solid recrystallized from pentane at $-35{ }^{\circ} \mathrm{C}$ yielding $0.009 \mathrm{~g}(71 \%)$ of $(\mathrm{BDI}-3) \operatorname{Ir}(\mathrm{CO})_{2}$. Anal. Calcd. for $\mathrm{C}_{31} \mathrm{H}_{41} \mathrm{IrN}_{2} \mathrm{O}_{2}$ : C, 55.92; H, 6.21; N, 4.21. Found: C, 55.44; H, 5.69; N, 3.94. ${ }^{1} \mathrm{H}$ NMR (benzene- $\left.d_{6}\right): \delta=1.08\left(\mathrm{~d}, 12 \mathrm{H}, 7 \mathrm{~Hz}, \mathrm{CHMe} e_{2}\right), 1.47\left(\mathrm{~d}, 12 \mathrm{H}, 7 \mathrm{~Hz}, \mathrm{CHMe} e_{2}\right), 1.64$ (s, 6H, $\mathrm{CH}_{3}$ ), 3.30 (sept, 4H, 7Hz, $\mathrm{CHMe}_{2}$ ), 5.24 (s, 1H, CH), 7.10 (br s, 4H, m-ArH), $7.11(\mathrm{br} \mathrm{s}, 2 \mathrm{H}, p-\mathrm{Ar} H) .{ }^{13} \mathrm{C}$ NMR (benzene- $\left.d_{8}\right): \delta=23.44,24.49(\mathrm{CHMe})$ ), $24.57(\mathrm{CMe})$, $28.05\left(\mathrm{CHMe}_{2}\right), 100.97(\mathrm{CH}), 124.14$ (p-Ar), $127.70(m-\mathrm{Ar}), 141.35$ (o-Ar), $154.62(\mathrm{~N}-$ C), $160.47(C \mathrm{Me}), 198.50(C \mathrm{O})$. IR (pentane): $v_{\mathrm{CO}}=1985 \mathrm{~cm}^{-1}, 2053 \mathrm{~cm}^{-1}$.

Preparation of (BDI-4) $\mathbf{I r}(\mathbf{C O})_{2}$. A thick walled glass vessel was charged with $0.014 \mathrm{~g}$ (0.017 mmol) of (BDI-4) $\operatorname{Ir}(\mathrm{COD})$ and approximately $15 \mathrm{~mL}$ of pentane. On the vacuum line, the vessel was submerged in liquid nitrogen, evacuated and one atmosphere of $\mathrm{CO}$ added. The contents of the vessel were thawed and the resulting solution stirred for 24 hours at ambient temperature. The pentane was removed in vacuo yielding $0.011 \mathrm{~g} \mathrm{(82 \% )}$ of a yellow powder identified as (BDI-4) $\operatorname{Ir}(\mathrm{CO})_{2}$. Anal. Calcd. for $\mathrm{C}_{31} \mathrm{H}_{35} \mathrm{IrN}_{2} \mathrm{O}_{2} \mathrm{~F}_{6}$ : C, 48.12; H, 4.56; N, 3.62. Found: C, 48.55; H, 5.01; N, 3.66. ${ }^{1} \mathrm{H}$ NMR (benzene- $d_{6}$ ): $\delta$ $=1.32\left(\mathrm{~d}, 12 \mathrm{H}, 7 \mathrm{~Hz}, \mathrm{CHMe} e_{2}\right), 1.60\left(\mathrm{~d}, 12 \mathrm{H}, 7 \mathrm{~Hz}, \mathrm{CHMe} e_{2}\right), 3.13\left(\mathrm{sept}, 4 \mathrm{H}, 7 \mathrm{~Hz}, \mathrm{CHMe} \mathrm{M}_{2}\right)$ 
$6.75(\mathrm{~s}, 1 \mathrm{H}, \mathrm{CH}), 7.15$ (br s, $4 \mathrm{H}, m-\mathrm{Ar} H$ ), 7.25 (br s, $2 \mathrm{H}, p-\mathrm{Ar} H$ ). ${ }^{13} \mathrm{C}$ NMR (benzene- $d_{8}$ ): $\delta=23.82,25.26\left(\mathrm{CHMe}_{2}\right), 29.15\left(\mathrm{CHMe}_{2}\right), 92.32(\mathrm{CH}), 123.82(p-\mathrm{Ar}), 128.88(m-\mathrm{Ar})$, $141.18(o-\operatorname{Ar}), 148.70\left(\mathrm{CF}_{3}\right), 153.76(\mathrm{~N}-C), 170.18(\mathrm{CO}) .{ }^{19} \mathrm{~F}$ NMR (benzene- $\left.d_{6}\right): \delta=$ $102.62\left(\mathrm{~s}, \mathrm{C} F_{3}\right)$. IR (pentane): $v_{\mathrm{CO}}=2005 \mathrm{~cm}^{-1}, 2069 \mathrm{~cm}^{-1}$.

Preparation of (BDI-1) IrH $\mathbf{I}_{2} \mathbf{N}_{2}$. A thick walled glass vessel was charged with $0.098 \mathrm{~g}$ $(0.15 \mathrm{mmol})$ of (BDI-1) $\operatorname{Ir}(\mathrm{COE}) \mathrm{N}_{2}$ and approximately $10 \mathrm{~mL}$ of pentane. On the vacuum line, the vessel was submerged in liquid nitrogen, evacuated and one atmosphere of $\mathrm{H}_{2}$ admitted. The contents of the vessel were then thawed and the resulting yellow solution stirred at ambient temperature for 8 hours. The resulting dark purple solution was transferrd into the dry box and filtered through a glass frit. The filtrate was collected and the pentane removed in vacuo yielding a dark foam. Recrystallization from pentane at $-35{ }^{\circ} \mathrm{C}$ yielded $0.024 \mathrm{~g}(30 \%)$ of a black solid identified as (BDI-1) $\operatorname{IrH}_{2} \mathrm{~N}_{2}$. Anal. Calcd. for CHIrN: C,; H,; N,. Found: C,; H,; N,. ${ }^{1} \mathrm{H}$ NMR (cyclohexane- $\left.d_{12}\right)$ : $\delta=1.59(\mathrm{~s}, 6 \mathrm{H}$, ArMe), 1.80 (s, 6H, ArMe), 2.18 (s, 3H, $\mathrm{CH}_{3}$ ), 2.26 (s, 3H, $\mathrm{CH}_{3}$ ), 5.36 (s, $\left.1 \mathrm{H}, \mathrm{CH}\right), 6.93-$ $7.08(\operatorname{Ar} H),-20.45(\mathrm{~s}, 2 \mathrm{H}, \mathrm{Ir}-H),-20.09\left(\mathrm{t}, 3.6 \mathrm{~Hz}, 1 \mathrm{H}, \mathrm{Ir}-H \mathrm{D}\right.$ in benzene- $\left.d_{6}\right) .{ }^{13} \mathrm{C}$ NMR $\left(\right.$ cyclohexane- $\left.d_{12}\right): \delta=19.03,19.27(\mathrm{ArMe}), 22.29,22.46(\mathrm{CMe}), 101.97(\mathrm{CH}), 124.76$, $125.85(p-A r), 128.41(m-A r), 130.91,131.51(o-A r) . \quad$ IR (benzene- $\left.d_{6}\right): v_{\mathrm{N}-\mathrm{N}}=2167 \mathrm{~cm}^{-1}$; IR (KBr): $v_{\mathrm{N}-\mathrm{N}}=2170 \mathrm{~cm}^{-1}$.

Thermolysis of (BDI-1) $\operatorname{Ir}(\mathbf{C O E}) \mathbf{N}_{2}$. A J. Young NMR tube was charged with $8 \mathrm{mg}$ $(0.013 \mathrm{mmol})$ of (BDI-1) $\operatorname{Ir}(\mathrm{COE}) \mathrm{N}_{2}$ and approximately $0.5 \mathrm{~mL}$ of cyclohexane- $d_{12}$. On the vacuum line, the tube was submerged in liquid nitrogen and evacuated. The contents of the tube were thawed and the procedure repeated. The resulting reaction mixture was 
thermolyzed in a $50{ }^{\circ} \mathrm{C}$ oil bath for 8 hours and monitored by ${ }^{1} \mathrm{H}$ NMR spectroscopy. The tube was periodically evacuated to remove the $\mathrm{N}_{2}$ product. The spectra collected over time revealed formation of (BDI-1) $\operatorname{Ir}\left(\eta^{3}-\mathrm{C}_{8} \mathrm{H}_{13}\right) \mathrm{H}$ followed by the appearance of (BDI1) $\mathrm{Ir}(\mathrm{COE}) \mathrm{H}_{2}$ and two irdium cyclooctadiene complexes in a 14:1 ratio. The major product was identified as (BDI-1) $\operatorname{Ir}(1,4-\mathrm{COD})$. (BDI-1) $\operatorname{Ir}\left(\mathbf{1 , 4 - C O D ) :}{ }^{1} \mathrm{H} \quad \mathrm{NMR}\right.$ (cyclohexane- $\left.d_{12}\right): \delta=1.47(\mathrm{~m}, 1,4-\mathrm{COD}), 1.52(\mathrm{~s}, 12 \mathrm{H}, \mathrm{ArMe}), 1.63\left(\mathrm{~s}, 6 \mathrm{H}, \mathrm{CH}_{3}\right), 1.94$ (m, 1,4-COD), 2.65 (m, 1,4-COD), 2.45 (m, 1,4-COD), 4.07 (dt, 2H, 1,4-COD), 5.32 (s, 1H, $C H), 6.82-7.11(\mathrm{Ar} H)$.

Preparation of 3. This molecule was prepared in a manner similar to 2 with $10 \mathrm{mg}(0.02$ mmol) of (BDI-2) $\operatorname{Ir}(\mathrm{COE}) \mathrm{N}_{2}$ in $0.5 \mathrm{~mL}$ of benzene- $d_{6}$ resulting in a red solution of $\mathbf{3}$ identified in approximately $85 \%$ yield by ${ }^{1} \mathrm{H}$ NMR spectroscopy. ${ }^{1} \mathrm{H}$ NMR (benzene- $d_{6}$ ): $\delta=1.15\left(\mathrm{t}, 3 \mathrm{H}, 12 \mathrm{~Hz}, \mathrm{CH}_{2} \mathrm{Me}\right), 1.25\left(\mathrm{t}, 3 \mathrm{H}, 12 \mathrm{~Hz}, \mathrm{CH}_{2} \mathrm{Me}\right), 1.26\left(\mathrm{t}, 3 \mathrm{H}, 12 \mathrm{~Hz}, \mathrm{CH}_{2} \mathrm{Me}\right)$, $1.67\left(\mathrm{~s}, 3 \mathrm{H}, \mathrm{CH}_{3}\right), 1.82\left(\mathrm{~s}, 3 \mathrm{H}, \mathrm{CH}_{3}\right), 2.26\left(\mathrm{~m}, 2 \mathrm{H}, \mathrm{CH}_{2} \mathrm{Me}\right), 2.48\left(\mathrm{~m}, 2 \mathrm{H}, \mathrm{CH}_{2} \mathrm{Me}\right), 2.75$ (m, 2H, $\mathrm{CH}_{2} \mathrm{Me}$ ), $4.12\left(\mathrm{dd}, 1 \mathrm{H},{ }^{l} J=11,{ }^{2} J=1.2 \mathrm{~Hz}\right.$, olefinic $\mathrm{CH}$ ), $4.25 \mathrm{dd}, 1 \mathrm{H},{ }^{l} J=11,{ }^{2} J=$ $1.2 \mathrm{~Hz}$, olefinic $\mathrm{CH}), 2.34(\mathrm{dd}, 1 \mathrm{H}$, olefinic $\mathrm{CH}), 4.78(\mathrm{~s}, 1 \mathrm{H}, \mathrm{CH}), 6.65-7.18(\mathrm{ArH})$.

Preparation of 4. This molecule was prepared in a similar manner to (BDI-1) $\operatorname{Ir}(\mathrm{CO})_{2}$ with approximately $10 \mathrm{mg}$ of $\mathbf{4}$ prepared in situ from (BDI-2) $\operatorname{Ir}(\mathrm{COE}) \mathrm{N}_{2}$ in approximately $0.5 \mathrm{~mL}$ of benzene- $d_{6}$. On the vacuum line, the solution was frozen at $77 \mathrm{~K}$ one atmosphere of $\mathrm{CO}$ was admitted. The solution was thawed and the resulting yellow solution was left to stand at ambient temperature for 1 day. ${ }^{1} \mathrm{H}$ NMR (benzene- $d_{6}$ ): $\delta=$ $1.24\left(\mathrm{t}, 3 \mathrm{H}, 12 \mathrm{~Hz}, \mathrm{CH}_{2} \mathrm{Me}\right), 1.26\left(\mathrm{t}, 3 \mathrm{H}, 12 \mathrm{~Hz}, \mathrm{CH}_{2} \mathrm{Me}\right), 1.27$ (t, 3H, 12Hz, $\left.\mathrm{CH}_{2} \mathrm{Me}\right), 1.51$ (s, 3H, $\left.\mathrm{CH}_{3}\right), 1.56\left(\mathrm{~s}, 3 \mathrm{H}, \mathrm{CH}_{3}\right), 2.53\left(\mathrm{~m}, 2 \mathrm{H}, \mathrm{CH}_{2} \mathrm{Me}\right), 2.59\left(\mathrm{~m}, 2 \mathrm{H}, \mathrm{CH}_{2} \mathrm{Me}\right), 2.96(\mathrm{~m}$, 
$\left.2 \mathrm{H}, \mathrm{CH}_{2} \mathrm{Me}\right), 5.11\left(\mathrm{dd}, 1 \mathrm{H},{ }^{1} \mathrm{~J}=17,{ }^{2} \mathrm{~J}=1.4 \mathrm{~Hz}\right.$, olefinic $\left.\mathrm{CH}\right), 5.18(\mathrm{dd}, 1 \mathrm{H}$, olefinic $\mathrm{CH})$, $5.61 \mathrm{dd}, 1 \mathrm{H},{ }^{1} J=17,{ }^{2} \mathrm{~J}=1.4 \mathrm{~Hz}$, olefinic $\left.\mathrm{CH}\right), 5.17(\mathrm{~s}, 1 \mathrm{H}, \mathrm{CH}), 6.98-7.41(\mathrm{ArH}) . \quad \mathrm{IR}$ (pentane): $v_{\mathrm{CO}}=2055 \mathrm{~cm}^{-1}, 1986 \mathrm{~cm}^{-1}$. 


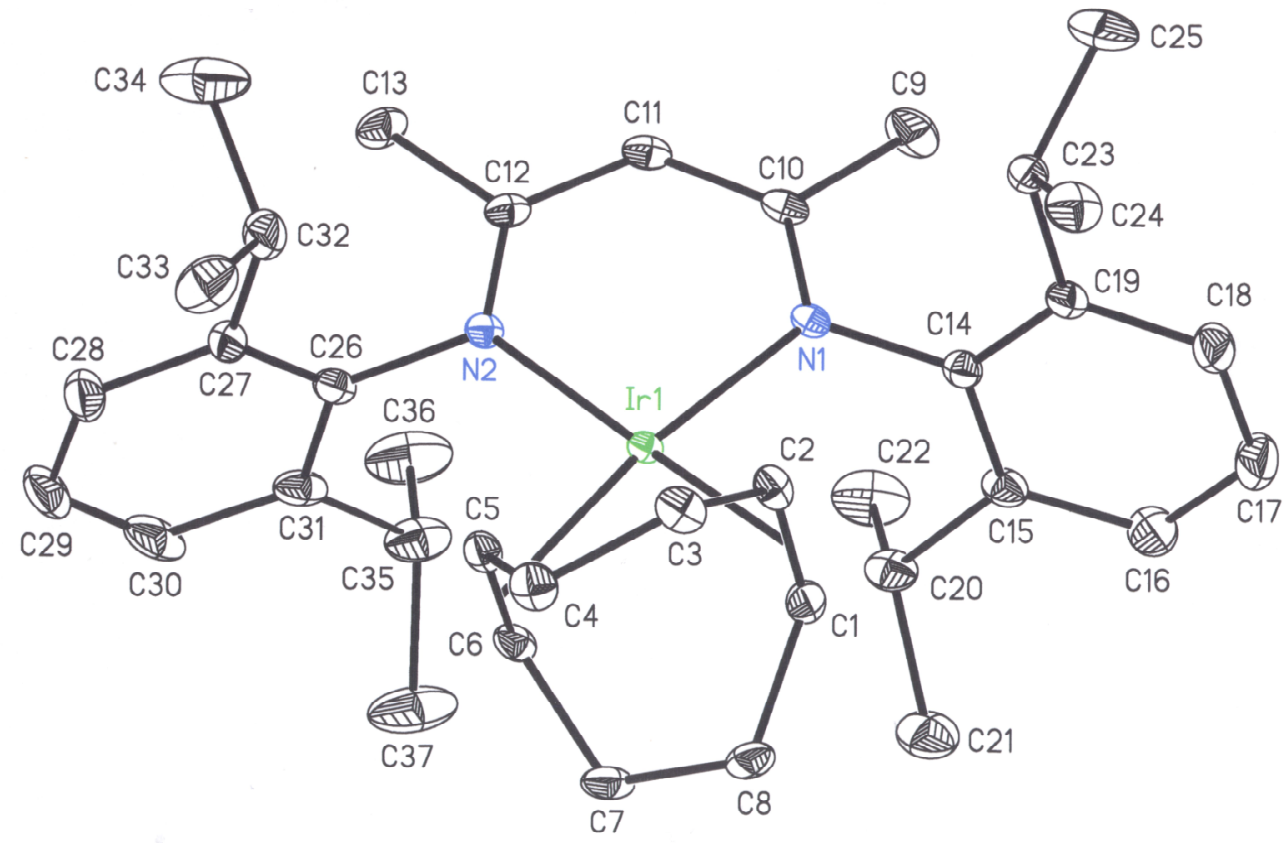

Figure S1. Fully labeled view of the molecular structure of (BDI-3) $\operatorname{Ir}(\mathrm{COD})$ at $30 \%$ probability ellipsoids. Hydrogen atoms omitted for clarity. 
Table S1. Crystal data and structure refinement for (BDI-3) $\operatorname{Ir}(\mathrm{COD})$.

Identification code

Empirical formula

Formula weight

Temperature

Wavelength

Crystal system

Space group

Unit cell dimensions

Volume

Z

Density (calculated)

Absorption coefficient

$\mathrm{F}(000)$

Crystal size

Theta range for data collection

Index ranges

Reflections collected

Independent reflections

Completeness to theta $=33.14^{\circ}$

Absorption correction

Max. and min. transmission

Refinement method

Data / restraints / parameters

Goodness-of-fit on $\mathrm{F}^{2}$

Final $\mathrm{R}$ indices [I $>2 \operatorname{sigma}(\mathrm{I})]$

$\mathrm{R}$ indices (all data)

Largest diff. peak and hole $\operatorname{cb} 3$

C37 H53 Ir N2

718.01

173(2) K

$0.71073 \AA$

Monoclinic

$\mathrm{C} 2 / \mathrm{c}$

$\begin{array}{ll}\mathrm{a}=33.2170(8) \AA & \alpha=90^{\circ} . \\ \mathrm{b}=13.0464(3) \AA & \beta=110.693(1)^{\circ} . \\ \mathrm{c}=16.0041(3) \AA & \gamma=90^{\circ} .\end{array}$

6488.1(2) $\AA^{3}$

8

$1.470 \mathrm{Mg} / \mathrm{m}^{3}$

$4.143 \mathrm{~mm}^{-1}$

2928

$0.30 \times 0.20 \times 0.15 \mathrm{~mm}^{3}$

1.31 to $33.14^{\circ}$.

$-50<=\mathrm{h}<=39,-19<=\mathrm{k}<=15,-23<=\mathrm{l}<=23$

37846

$10890[\mathrm{R}(\mathrm{int})=0.0464]$

$88.0 \%$

\section{SADABS}

0.5754 and 0.3696

Full-matrix least-squares on $\mathrm{F}^{2}$

$10890 / 0 / 361$

0.999

$\mathrm{R} 1=0.0337, \mathrm{wR} 2=0.0712$

$\mathrm{R} 1=0.0544, \mathrm{wR} 2=0.0795$

2.298 and -1.570 e. $\AA^{-3}$ 
Table S2. Bond lengths $[\AA]$ and angles $\left[^{\circ}\right]$ for (BDI-3) Ir(COD).

\begin{tabular}{|c|c|c|c|}
\hline $\operatorname{Ir}(1)-\mathrm{N}(2)$ & $2.088(2)$ & $C(26)-C(27)$ & $1.404(5)$ \\
\hline $\operatorname{Ir}(1)-\mathrm{N}(1)$ & $2.096(2)$ & $C(26)-C(31)$ & $1.419(5)$ \\
\hline $\operatorname{Ir}(1)-C(6)$ & $2.116(3)$ & $C(27)-C(28)$ & $1.390(5)$ \\
\hline $\operatorname{Ir}(1)-C(2)$ & $2.117(3)$ & $C(27)-C(32)$ & $1.528(5)$ \\
\hline $\operatorname{Ir}(1)-C(5)$ & $2.143(3)$ & $C(28)-C(29)$ & $1.391(6)$ \\
\hline $\operatorname{Ir}(1)-C(1)$ & $2.165(3)$ & $C(29)-C(30)$ & $1.378(7)$ \\
\hline $\mathrm{N}(1)-\mathrm{C}(10)$ & $1.352(3)$ & $C(30)-C(31)$ & $1.396(5)$ \\
\hline $\mathrm{N}(1)-\mathrm{C}(14)$ & $1.443(4)$ & $C(31)-C(35)$ & $1.522(6)$ \\
\hline $\mathrm{N}(2)-\mathrm{C}(12)$ & $1.340(4)$ & $C(32)-C(33)$ & $1.525(6)$ \\
\hline $\mathrm{N}(2)-\mathrm{C}(26)$ & $1.444(4)$ & $C(32)-C(34)$ & $1.523(6)$ \\
\hline$C(1)-C(2)$ & $1.413(5)$ & $C(35)-C(36)$ & $1.532(6)$ \\
\hline $\mathrm{C}(1)-\mathrm{C}(8)$ & $1.513(4)$ & $C(35)-C(37)$ & $1.542(6)$ \\
\hline $\mathrm{C}(2)-\mathrm{C}(3)$ & $1.514(4)$ & & \\
\hline$C(3)-C(4)$ & $1.532(5)$ & $\mathrm{N}(2)-\operatorname{Ir}(1)-\mathrm{N}(1)$ & $90.14(10)$ \\
\hline$C(4)-C(5)$ & $1.529(4)$ & $\mathrm{N}(2)-\operatorname{Ir}(1)-\mathrm{C}(6)$ & $93.25(11)$ \\
\hline$C(5)-C(6)$ & $1.414(5)$ & $\mathrm{N}(1)-\operatorname{Ir}(1)-\mathrm{C}(6)$ & $151.12(12)$ \\
\hline$C(6)-C(7)$ & $1.513(5)$ & $\mathrm{N}(2)-\operatorname{Ir}(1)-\mathrm{C}(2)$ & $152.57(12)$ \\
\hline$C(7)-C(8)$ & $1.528(5)$ & $\mathrm{N}(1)-\operatorname{Ir}(1)-\mathrm{C}(2)$ & $93.36(11)$ \\
\hline $\mathrm{C}(9)-\mathrm{C}(10)$ & $1.512(4)$ & $C(6)-\operatorname{Ir}(1)-C(2)$ & $96.62(12)$ \\
\hline C(10)-C(11) & $1.383(5)$ & $\mathrm{N}(2)-\operatorname{Ir}(1)-\mathrm{C}(5)$ & $92.32(11)$ \\
\hline C(11)-C(12) & $1.392(5)$ & $\mathrm{N}(1)-\operatorname{Ir}(1)-\mathrm{C}(5)$ & $169.57(11)$ \\
\hline$C(12)-C(13)$ & $1.519(4)$ & $\mathrm{C}(6)-\operatorname{Ir}(1)-\mathrm{C}(5)$ & $38.79(13)$ \\
\hline C(14)-C(19) & $1.410(5)$ & $C(2)-\operatorname{Ir}(1)-C(5)$ & $79.86(12)$ \\
\hline C(14)-C(15) & $1.409(4)$ & $\mathrm{N}(2)-\operatorname{Ir}(1)-\mathrm{C}(1)$ & $168.54(12)$ \\
\hline C(15)-C(16) & $1.390(5)$ & $\mathrm{N}(1)-\operatorname{Ir}(1)-\mathrm{C}(1)$ & $91.69(11)$ \\
\hline C(15)-C(20) & $1.529(5)$ & $\mathrm{C}(6)-\operatorname{Ir}(1)-\mathrm{C}(1)$ & $79.78(12)$ \\
\hline C(16)-C(17) & $1.389(6)$ & $\mathrm{C}(2)-\operatorname{Ir}(1)-\mathrm{C}(1)$ & $38.53(13)$ \\
\hline C(17)-C(18) & $1.374(6)$ & $C(5)-\operatorname{Ir}(1)-C(1)$ & $87.90(12)$ \\
\hline C(18)-C(19) & $1.398(5)$ & $\mathrm{C}(10)-\mathrm{N}(1)-\mathrm{C}(14)$ & $114.4(2)$ \\
\hline C(19)-C(23) & $1.524(5)$ & $C(10)-N(1)-\operatorname{Ir}(1)$ & $124.5(2)$ \\
\hline $\mathrm{C}(20)-\mathrm{C}(21)$ & $1.520(5)$ & $\mathrm{C}(14)-\mathrm{N}(1)-\operatorname{Ir}(1)$ & $120.18(18)$ \\
\hline$C(20)-C(22)$ & $1.530(5)$ & $\mathrm{C}(12)-\mathrm{N}(2)-\mathrm{C}(26)$ & $114.0(2)$ \\
\hline$C(23)-C(24)$ & $1.525(5)$ & $\mathrm{C}(12)-\mathrm{N}(2)-\operatorname{Ir}(1)$ & $125.6(2)$ \\
\hline$C(23)-C(25)$ & $1.536(5)$ & $\mathrm{C}(26)-\mathrm{N}(2)-\operatorname{Ir}(1)$ & $120.20(17)$ \\
\hline
\end{tabular}




\begin{tabular}{|c|c|}
\hline $\mathrm{C}(2)-\mathrm{C}(1)-\mathrm{C}(8)$ & $123.4(3)$ \\
\hline$C(2)-C(1)-\operatorname{Ir}(1)$ & $68.91(17)$ \\
\hline$C(8)-C(1)-\operatorname{Ir}(1)$ & $114.2(2)$ \\
\hline$C(1)-C(2)-C(3)$ & $124.3(3)$ \\
\hline$C(1)-C(2)-\operatorname{Ir}(1)$ & $72.57(17)$ \\
\hline$C(3)-C(2)-\operatorname{Ir}(1)$ & $113.1(2)$ \\
\hline$C(2)-C(3)-C(4)$ & $112.0(3)$ \\
\hline$C(5)-C(4)-C(3)$ & $110.9(2)$ \\
\hline$C(6)-C(5)-C(4)$ & $124.3(3)$ \\
\hline$C(6)-C(5)-\operatorname{Ir}(1)$ & $69.60(18)$ \\
\hline$C(4)-C(5)-\operatorname{Ir}(1)$ & $115.2(2)$ \\
\hline$C(5)-C(6)-C(7)$ & $125.4(3)$ \\
\hline$C(5)-C(6)-\operatorname{Ir}(1)$ & 71.61(18) \\
\hline$C(7)-C(6)-\operatorname{Ir}(1)$ & $112.4(2)$ \\
\hline $\mathrm{C}(6)-\mathrm{C}(7)-\mathrm{C}(8)$ & $111.4(3)$ \\
\hline$C(1)-C(8)-C(7)$ & $111.2(2)$ \\
\hline $\mathrm{N}(1)-\mathrm{C}(10)-\mathrm{C}(11)$ & $124.9(3)$ \\
\hline $\mathrm{N}(1)-\mathrm{C}(10)-\mathrm{C}(9)$ & $120.2(3)$ \\
\hline $\mathrm{C}(11)-\mathrm{C}(10)-\mathrm{C}(9)$ & $114.9(3)$ \\
\hline $\mathrm{C}(10)-\mathrm{C}(11)-\mathrm{C}(12)$ & $129.7(3)$ \\
\hline $\mathrm{N}(2)-\mathrm{C}(12)-\mathrm{C}(11)$ & $124.1(3)$ \\
\hline $\mathrm{N}(2)-\mathrm{C}(12)-\mathrm{C}(13)$ & $121.8(3)$ \\
\hline $\mathrm{C}(11)-\mathrm{C}(12)-\mathrm{C}(13)$ & 114.1(3) \\
\hline $\mathrm{C}(19)-\mathrm{C}(14)-\mathrm{C}(15)$ & $120.5(3)$ \\
\hline $\mathrm{C}(19)-\mathrm{C}(14)-\mathrm{N}(1)$ & $121.6(3)$ \\
\hline $\mathrm{C}(15)-\mathrm{C}(14)-\mathrm{N}(1)$ & $117.8(3)$ \\
\hline$C(16)-C(15)-C(14)$ & $118.6(3)$ \\
\hline $\mathrm{C}(16)-\mathrm{C}(15)-\mathrm{C}(20)$ & $120.3(3)$ \\
\hline$C(14)-C(15)-C(20)$ & $121.1(3)$ \\
\hline $\mathrm{C}(17)-\mathrm{C}(16)-\mathrm{C}(15)$ & $121.3(4)$ \\
\hline $\mathrm{C}(18)-\mathrm{C}(17)-\mathrm{C}(16)$ & $119.8(4)$ \\
\hline $\mathrm{C}(17)-\mathrm{C}(18)-\mathrm{C}(19)$ & $121.2(4)$ \\
\hline
\end{tabular}

$\begin{array}{ll}\mathrm{C}(18)-\mathrm{C}(19)-\mathrm{C}(14) & 118.6(3) \\ \mathrm{C}(18)-\mathrm{C}(19)-\mathrm{C}(23) & 118.2(3) \\ \mathrm{C}(14)-\mathrm{C}(19)-\mathrm{C}(23) & 123.2(3) \\ \mathrm{C}(21)-\mathrm{C}(20)-\mathrm{C}(15) & 112.8(3) \\ \mathrm{C}(21)-\mathrm{C}(20)-\mathrm{C}(22) & 109.7(3) \\ \mathrm{C}(15)-\mathrm{C}(20)-\mathrm{C}(22) & 112.1(3) \\ \mathrm{C}(19)-\mathrm{C}(23)-\mathrm{C}(24) & 112.3(3) \\ \mathrm{C}(19)-\mathrm{C}(23)-\mathrm{C}(25) & 111.8(3) \\ \mathrm{C}(24)-\mathrm{C}(23)-\mathrm{C}(25) & 107.3(3) \\ \mathrm{C}(27)-\mathrm{C}(26)-\mathrm{C}(31) & 121.5(3) \\ \mathrm{C}(27)-\mathrm{C}(26)-\mathrm{N}(2) & 121.1(3) \\ \mathrm{C}(31)-\mathrm{C}(26)-\mathrm{N}(2) & 117.4(3) \\ \mathrm{C}(28)-\mathrm{C}(27)-\mathrm{C}(26) & 118.2(4) \\ \mathrm{C}(28)-\mathrm{C}(27)-\mathrm{C}(32) & 120.0(4) \\ \mathrm{C}(26)-\mathrm{C}(27)-\mathrm{C}(32) & 121.7(3) \\ \mathrm{C}(29)-\mathrm{C}(28)-\mathrm{C}(27) & 121.4(4) \\ \mathrm{C}(30)-\mathrm{C}(29)-\mathrm{C}(28) & 119.5(4) \\ \mathrm{C}(29)-\mathrm{C}(30)-\mathrm{C}(31) & 122.0(4) \\ \mathrm{C}(30)-\mathrm{C}(31)-\mathrm{C}(26) & 117.3(4) \\ \mathrm{C}(30)-\mathrm{C}(31)-\mathrm{C}(35) & 121.5(4) \\ \mathrm{C}(26)-\mathrm{C}(31)-\mathrm{C}(35) & 121.2(3) \\ \mathrm{C}(33)-\mathrm{C}(32)-\mathrm{C}(34) & 108.6(3) \\ \mathrm{C}(33)-\mathrm{C}(32)-\mathrm{C}(27) & 113.3(3) \\ \mathrm{C}(34)-\mathrm{C}(32)-\mathrm{C}(27) & 111.5(4) \\ \mathrm{C}(36)-\mathrm{C}(35)-\mathrm{C}(31) & 112.8(3) \\ \mathrm{C}(36)-\mathrm{C}(35)-\mathrm{C}(37) & 109.4(3) \\ \mathrm{C}(31)-\mathrm{C}(35)-\mathrm{C}(37) & 112.3(4) \\ & \\ & \end{array}$

Symmetry transformations used to generate equivalent atoms: 


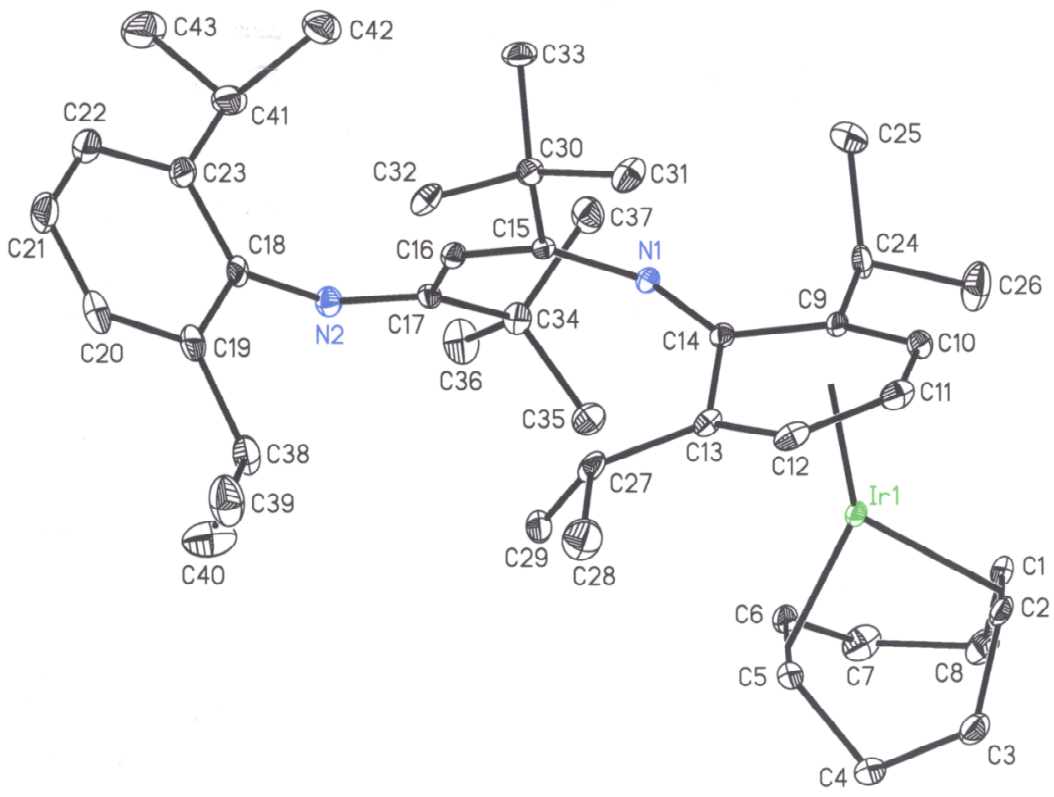

Figure S2. Fully labeled view of the molecular structure of (BDI-5) $\operatorname{Ir}(\mathrm{COD})$ at $30 \%$ probability ellipsoids. Hydrogen atoms omitted for clarity. 
Table S3. Crystal data and structure refinement for (BDI-5) $\operatorname{Ir}(\mathrm{COD})$.

Identification code

Empirical formula

Formula weight

Temperature

Wavelength

Crystal system

Space group

Unit cell dimensions

Volume

Z

Density (calculated)

Absorption coefficient

$\mathrm{F}(000)$

Crystal size

Theta range for data collection

Index ranges

Reflections collected

Independent reflections

Completeness to theta $=30.51^{\circ}$

Absorption correction

Max. and min. transmission

Refinement method

Data / restraints / parameters

Goodness-of-fit on $\mathrm{F}^{2}$

Final $\mathrm{R}$ indices $[\mathrm{I}>2 \operatorname{sigma}(\mathrm{I})]$

$\mathrm{R}$ indices (all data)

Largest diff. peak and hole wb5

C43 H65 Ir N2

802.17

173(2) K

$0.71073 \AA$

Monoclinic

$\mathrm{P} 2(1) / \mathrm{n}$

$\mathrm{a}=11.8294(10) \AA$

$\alpha=90^{\circ}$.

$\mathrm{b}=19.1650(17) \AA$

$\beta=91.796(2)^{\circ}$.

$\mathrm{c}=16.9639(15) \AA$

$\gamma=90^{\circ}$.

4

$1.386 \mathrm{Mg} / \mathrm{m}^{3}$

$3.504 \mathrm{~mm}^{-1}$

1656

$0.25 \times 0.05 \times 0.03 \mathrm{~mm}^{3}$

2.07 to $30.51^{\circ}$.

$-16<=\mathrm{h}<=16,-26<=\mathrm{k}<=26,-23<=\mathrm{l}<=23$

44018

$11320[\mathrm{R}(\mathrm{int})=0.0782]$

$96.5 \%$

\section{SADABS}

0.9175 and 0.4745

Full-matrix least-squares on $\mathrm{F}^{2}$

$11320 / 0 / 651$

0.953

$\mathrm{R} 1=0.0371, \mathrm{wR} 2=0.0701$

$\mathrm{R} 1=0.0738, \mathrm{wR} 2=0.0807$

1.307 and -0.841 e. $\AA^{-3}$ 
Table S4. Bond lengths $[\AA]$ and angles $\left[{ }^{\circ}\right]$ for (BDI-5) $\operatorname{Ir}(\mathrm{COD})$.

\begin{tabular}{|c|c|c|c|}
\hline $\operatorname{Ir}(1)-C(5)$ & $2.108(4)$ & $C(18)-C(19)$ & $1.418(5)$ \\
\hline $\operatorname{Ir}(1)-C(6)$ & $2.113(4)$ & $C(19)-C(20)$ & $1.383(6)$ \\
\hline $\operatorname{Ir}(1)-C(1)$ & $2.137(4)$ & $C(19)-C(38)$ & $1.522(6)$ \\
\hline $\operatorname{Ir}(1)-C(2)$ & $2.142(4)$ & $C(20)-C(21)$ & $1.400(7)$ \\
\hline $\operatorname{Ir}(1)-C(10)$ & $2.231(4)$ & $C(21)-C(22)$ & $1.363(7)$ \\
\hline $\operatorname{Ir}(1)-C(11)$ & $2.241(4)$ & $C(22)-C(23)$ & $1.386(6)$ \\
\hline $\operatorname{Ir}(1)-C(12)$ & $2.245(4)$ & $C(23)-C(41)$ & $1.518(6)$ \\
\hline $\operatorname{Ir}(1)-C(13)$ & $2.292(4)$ & $C(24)-C(26)$ & $1.521(6)$ \\
\hline $\operatorname{Ir}(1)-C(9)$ & $2.373(4)$ & $C(24)-C(25)$ & $1.532(6)$ \\
\hline $\mathrm{N}(1)-\mathrm{C}(14)$ & $1.295(4)$ & $C(27)-C(29)$ & $1.517(6)$ \\
\hline $\mathrm{N}(1)-\mathrm{C}(15)$ & $1.366(4)$ & $C(27)-C(28)$ & $1.519(6)$ \\
\hline $\mathrm{N}(2)-\mathrm{C}(17)$ & $1.288(4)$ & $C(30)-C(32)$ & $1.538(5)$ \\
\hline $\mathrm{N}(2)-\mathrm{C}(18)$ & $1.418(5)$ & $C(30)-C(31)$ & $1.532(5)$ \\
\hline$C(1)-C(2)$ & $1.431(6)$ & $C(30)-C(33)$ & $1.537(5)$ \\
\hline $\mathrm{C}(1)-\mathrm{C}(8)$ & $1.516(6)$ & $C(34)-C(35)$ & $1.527(6)$ \\
\hline $\mathrm{C}(2)-\mathrm{C}(3)$ & $1.504(6)$ & $C(34)-C(37)$ & $1.531(6)$ \\
\hline$C(3)-C(4)$ & $1.526(6)$ & $C(34)-C(36)$ & $1.543(6)$ \\
\hline$C(4)-C(5)$ & $1.529(5)$ & $C(38)-C(40)$ & $1.524(7)$ \\
\hline$C(5)-C(6)$ & $1.426(6)$ & $\mathrm{C}(38)-\mathrm{C}(39)$ & $1.527(6)$ \\
\hline$C(6)-C(7)$ & $1.511(6)$ & $C(41)-C(43)$ & $1.521(6)$ \\
\hline$C(7)-C(8)$ & $1.536(6)$ & $\mathrm{C}(41)-\mathrm{C}(42)$ & $1.536(6)$ \\
\hline$C(9)-C(10)$ & $1.413(5)$ & & \\
\hline$C(9)-C(14)$ & $1.468(5)$ & $C(5)-\operatorname{Ir}(1)-C(6)$ & $39.50(15)$ \\
\hline $\mathrm{C}(9)-\mathrm{C}(24)$ & $1.516(5)$ & $C(5)-\operatorname{Ir}(1)-C(1)$ & $88.62(16)$ \\
\hline$C(10)-C(11)$ & $1.404(6)$ & $C(6)-\operatorname{Ir}(1)-C(1)$ & $80.30(16)$ \\
\hline $\mathrm{C}(11)-\mathrm{C}(12)$ & $1.399(6)$ & $C(5)-\operatorname{Ir}(1)-C(2)$ & $80.19(15)$ \\
\hline$C(12)-C(13)$ & $1.434(5)$ & $C(6)-\operatorname{Ir}(1)-C(2)$ & $97.52(15)$ \\
\hline$C(13)-C(14)$ & $1.479(5)$ & $\mathrm{C}(1)-\operatorname{Ir}(1)-\mathrm{C}(2)$ & $39.07(16)$ \\
\hline$C(13)-C(27)$ & $1.534(5)$ & $C(5)-\operatorname{Ir}(1)-C(10)$ & $175.04(16)$ \\
\hline$C(15)-C(16)$ & $1.372(5)$ & $C(6)-\operatorname{Ir}(1)-C(10)$ & $144.60(15)$ \\
\hline$C(15)-C(30)$ & $1.567(5)$ & $C(1)-\operatorname{Ir}(1)-C(10)$ & $94.96(16)$ \\
\hline$C(16)-C(17)$ & $1.464(5)$ & $C(2)-\operatorname{Ir}(1)-C(10)$ & $100.45(15)$ \\
\hline $\mathrm{C}(17)-\mathrm{C}(34)$ & $1.543(5)$ & $C(5)-\operatorname{Ir}(1)-C(11)$ & $138.63(16)$ \\
\hline$C(18)-C(23)$ & $1.414(5)$ & $C(6)-\operatorname{Ir}(1)-C(11)$ & $168.76(15)$ \\
\hline
\end{tabular}




\begin{tabular}{|c|c|c|c|}
\hline$C(1)-\operatorname{Ir}(1)-C(11)$ & $110.86(16)$ & $\mathrm{C}(4)-\mathrm{C}(5)-\operatorname{Ir}(1)$ & $115.6(3)$ \\
\hline$C(2)-\operatorname{Ir}(1)-C(11)$ & $92.39(15)$ & $\mathrm{C}(5)-\mathrm{C}(6)-\mathrm{C}(7)$ & $123.5(4)$ \\
\hline$C(10)-\operatorname{Ir}(1)-C(11)$ & $36.60(15)$ & $\mathrm{C}(5)-\mathrm{C}(6)-\operatorname{Ir}(1)$ & $70.1(2)$ \\
\hline$C(5)-\operatorname{Ir}(1)-C(12)$ & $109.68(16)$ & $C(7)-C(6)-\operatorname{Ir}(1)$ & 113.1(3) \\
\hline$C(6)-\operatorname{Ir}(1)-C(12)$ & $133.41(16)$ & $\mathrm{C}(6)-\mathrm{C}(7)-\mathrm{C}(8)$ & $111.8(4)$ \\
\hline$C(1)-\operatorname{Ir}(1)-C(12)$ & $143.84(16)$ & $\mathrm{C}(1)-\mathrm{C}(8)-\mathrm{C}(7)$ & 111.1(3) \\
\hline$C(2)-\operatorname{Ir}(1)-C(12)$ & $111.75(15)$ & $C(10)-C(9)-C(14)$ & $117.6(3)$ \\
\hline C(10)-Ir(1)-C(12) & $65.47(16)$ & $\mathrm{C}(10)-\mathrm{C}(9)-\mathrm{C}(24)$ & $121.0(3)$ \\
\hline C(11)-Ir(1)-C(12) & $36.34(16)$ & $\mathrm{C}(14)-\mathrm{C}(9)-\mathrm{C}(24)$ & $119.0(3)$ \\
\hline$C(5)-\operatorname{Ir}(1)-C(13)$ & $100.51(14)$ & $C(10)-C(9)-\operatorname{Ir}(1)$ & $66.7(2)$ \\
\hline$C(6)-\operatorname{Ir}(1)-C(13)$ & $103.48(14)$ & $C(14)-C(9)-\operatorname{Ir}(1)$ & $86.0(2)$ \\
\hline$C(1)-\operatorname{Ir}(1)-C(13)$ & $169.54(14)$ & $C(24)-C(9)-\operatorname{Ir}(1)$ & $131.7(3)$ \\
\hline$C(2)-\operatorname{Ir}(1)-C(13)$ & $147.29(15)$ & $\mathrm{C}(11)-\mathrm{C}(10)-\mathrm{C}(9)$ & $122.2(4)$ \\
\hline C(10)-Ir(1)-C(13) & $76.26(14)$ & $C(11)-C(10)-\operatorname{Ir}(1)$ & $72.1(2)$ \\
\hline C(11)-Ir(1)-C(13) & $65.30(14)$ & $C(9)-C(10)-\operatorname{Ir}(1)$ & $77.7(2)$ \\
\hline C(12)-Ir(1)-C(13) & $36.83(13)$ & $\mathrm{C}(12)-\mathrm{C}(11)-\mathrm{C}(10)$ & $119.4(4)$ \\
\hline$C(5)-\operatorname{Ir}(1)-C(9)$ & $146.09(13)$ & $\mathrm{C}(12)-\mathrm{C}(11)-\operatorname{Ir}(1)$ & $72.0(2)$ \\
\hline$C(6)-\operatorname{Ir}(1)-C(9)$ & $111.96(14)$ & $\mathrm{C}(10)-\mathrm{C}(11)-\operatorname{Ir}(1)$ & $71.3(2)$ \\
\hline$C(1)-\operatorname{Ir}(1)-C(9)$ & $106.21(14)$ & $\mathrm{C}(11)-\mathrm{C}(12)-\mathrm{C}(13)$ & $119.4(4)$ \\
\hline$C(2)-\operatorname{Ir}(1)-C(9)$ & $130.09(14)$ & $\mathrm{C}(11)-\mathrm{C}(12)-\operatorname{Ir}(1)$ & $71.7(2)$ \\
\hline$C(10)-\operatorname{Ir}(1)-C(9)$ & $35.57(13)$ & $C(13)-C(12)-\operatorname{Ir}(1)$ & $73.4(2)$ \\
\hline$C(11)-\operatorname{Ir}(1)-C(9)$ & $64.53(14)$ & $\mathrm{C}(12)-\mathrm{C}(13)-\mathrm{C}(14)$ & $118.3(3)$ \\
\hline$C(12)-\operatorname{Ir}(1)-C(9)$ & $76.06(14)$ & $\mathrm{C}(12)-\mathrm{C}(13)-\mathrm{C}(27)$ & $119.4(3)$ \\
\hline$C(13)-\operatorname{Ir}(1)-C(9)$ & $63.33(12)$ & $C(14)-C(13)-C(27)$ & $118.8(3)$ \\
\hline $\mathrm{C}(14)-\mathrm{N}(1)-\mathrm{C}(15)$ & $133.0(3)$ & $C(12)-C(13)-\operatorname{Ir}(1)$ & $69.8(2)$ \\
\hline $\mathrm{C}(17)-\mathrm{N}(2)-\mathrm{C}(18)$ & $123.2(3)$ & $C(14)-C(13)-\operatorname{Ir}(1)$ & $88.9(2)$ \\
\hline $\mathrm{C}(2)-\mathrm{C}(1)-\mathrm{C}(8)$ & $122.5(4)$ & $C(27)-C(13)-\operatorname{Ir}(1)$ & $128.9(3)$ \\
\hline$C(2)-C(1)-\operatorname{Ir}(1)$ & $70.7(2)$ & $\mathrm{N}(1)-\mathrm{C}(14)-\mathrm{C}(9)$ & $118.3(3)$ \\
\hline$C(8)-C(1)-\operatorname{Ir}(1)$ & $115.0(3)$ & $\mathrm{N}(1)-\mathrm{C}(14)-\mathrm{C}(13)$ & $128.7(3)$ \\
\hline$C(1)-C(2)-C(3)$ & $124.3(4)$ & $C(9)-C(14)-C(13)$ & $112.5(3)$ \\
\hline$C(1)-C(2)-\operatorname{Ir}(1)$ & $70.3(2)$ & $\mathrm{N}(1)-\mathrm{C}(15)-\mathrm{C}(16)$ & $125.4(3)$ \\
\hline$C(3)-C(2)-\operatorname{Ir}(1)$ & $112.2(3)$ & $\mathrm{N}(1)-\mathrm{C}(15)-\mathrm{C}(30)$ & $117.0(3)$ \\
\hline$C(2)-C(3)-C(4)$ & $112.2(3)$ & $C(16)-C(15)-C(30)$ & $115.9(3)$ \\
\hline$C(5)-C(4)-C(3)$ & 111.1(3) & $C(15)-C(16)-C(17)$ & $137.2(4)$ \\
\hline$C(6)-C(5)-C(4)$ & $123.4(4)$ & $\mathrm{N}(2)-\mathrm{C}(17)-\mathrm{C}(16)$ & $119.6(3)$ \\
\hline$C(6)-C(5)-\operatorname{Ir}(1)$ & $70.4(2)$ & $\mathrm{N}(2)-\mathrm{C}(17)-\mathrm{C}(34)$ & $115.2(3)$ \\
\hline
\end{tabular}




\begin{tabular}{|c|c|c|c|}
\hline $\mathrm{C}(16)-\mathrm{C}(17)-\mathrm{C}(34)$ & $124.7(3)$ & $\mathrm{C}(32)-\mathrm{C}(30)-\mathrm{C}(31)$ & $107.8(3)$ \\
\hline $\mathrm{C}(23)-\mathrm{C}(18)-\mathrm{C}(19)$ & $120.4(4)$ & $\mathrm{C}(32)-\mathrm{C}(30)-\mathrm{C}(33)$ & $108.8(3)$ \\
\hline $\mathrm{C}(23)-\mathrm{C}(18)-\mathrm{N}(2)$ & $117.0(3)$ & $\mathrm{C}(31)-\mathrm{C}(30)-\mathrm{C}(33)$ & $107.7(3)$ \\
\hline $\mathrm{C}(19)-\mathrm{C}(18)-\mathrm{N}(2)$ & $121.9(3)$ & $C(32)-C(30)-C(15)$ & $112.1(3)$ \\
\hline$C(20)-C(19)-C(18)$ & $118.4(4)$ & $\mathrm{C}(31)-\mathrm{C}(30)-\mathrm{C}(15)$ & $113.6(3)$ \\
\hline $\mathrm{C}(20)-\mathrm{C}(19)-\mathrm{C}(38)$ & $121.5(4)$ & $C(33)-C(30)-C(15)$ & $106.7(3)$ \\
\hline $\mathrm{C}(18)-\mathrm{C}(19)-\mathrm{C}(38)$ & $119.9(4)$ & $C(35)-C(34)-C(37)$ & $110.6(3)$ \\
\hline$C(19)-C(20)-C(21)$ & $120.8(4)$ & $C(35)-C(34)-C(17)$ & $112.6(3)$ \\
\hline$C(22)-C(21)-C(20)$ & $120.3(4)$ & $C(37)-C(34)-C(17)$ & $107.8(3)$ \\
\hline $\mathrm{C}(21)-\mathrm{C}(22)-\mathrm{C}(23)$ & $121.5(4)$ & $C(35)-C(34)-C(36)$ & $107.0(4)$ \\
\hline $\mathrm{C}(22)-\mathrm{C}(23)-\mathrm{C}(18)$ & $118.6(4)$ & $\mathrm{C}(37)-\mathrm{C}(34)-\mathrm{C}(36)$ & $108.6(4)$ \\
\hline $\mathrm{C}(22)-\mathrm{C}(23)-\mathrm{C}(41)$ & $122.0(4)$ & $\mathrm{C}(17)-\mathrm{C}(34)-\mathrm{C}(36)$ & $110.2(3)$ \\
\hline $\mathrm{C}(18)-\mathrm{C}(23)-\mathrm{C}(41)$ & 119.3(4) & $\mathrm{C}(19)-\mathrm{C}(38)-\mathrm{C}(40)$ & $110.3(4)$ \\
\hline$C(9)-C(24)-C(26)$ & $113.2(4)$ & $\mathrm{C}(19)-\mathrm{C}(38)-\mathrm{C}(39)$ & $114.0(4)$ \\
\hline$C(9)-C(24)-C(25)$ & $110.6(4)$ & $\mathrm{C}(40)-\mathrm{C}(38)-\mathrm{C}(39)$ & $110.2(4)$ \\
\hline $\mathrm{C}(26)-\mathrm{C}(24)-\mathrm{C}(25)$ & $110.2(4)$ & $C(23)-C(41)-C(43)$ & $114.1(4)$ \\
\hline $\mathrm{C}(29)-\mathrm{C}(27)-\mathrm{C}(28)$ & $108.8(4)$ & $\mathrm{C}(23)-\mathrm{C}(41)-\mathrm{C}(42)$ & $111.1(4)$ \\
\hline$C(29)-C(27)-C(13)$ & $114.5(3)$ & $C(43)-C(41)-C(42)$ & $109.5(4)$ \\
\hline $\mathrm{C}(28)-\mathrm{C}(27)-\mathrm{C}(13)$ & $114.6(4)$ & & \\
\hline
\end{tabular}




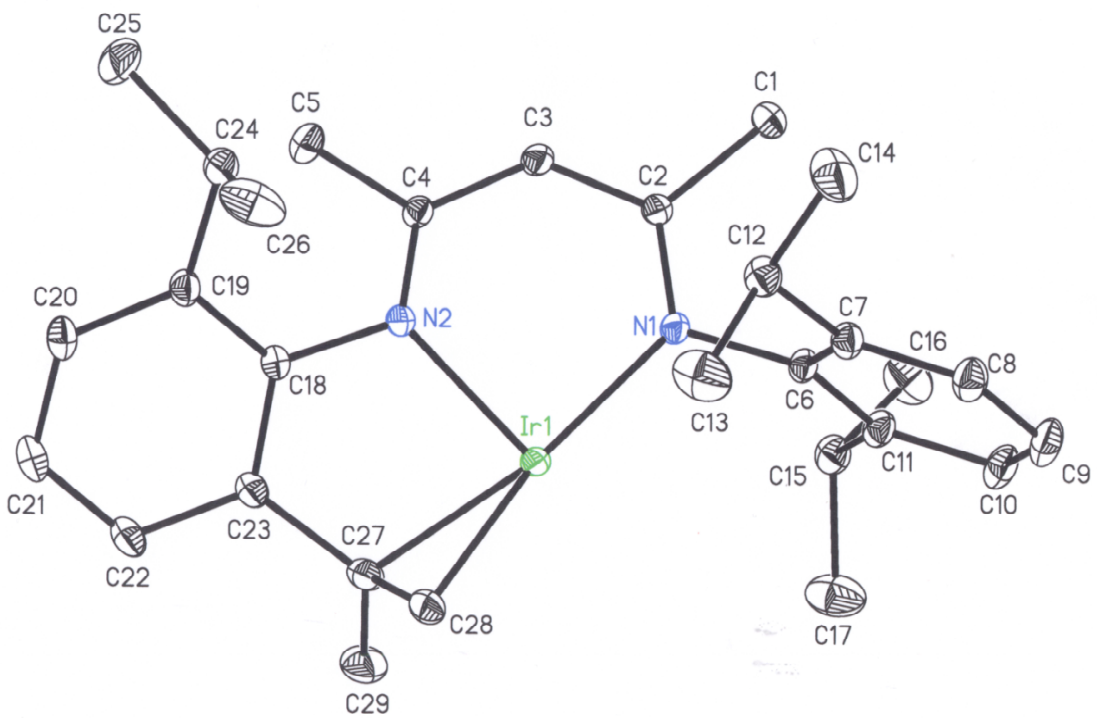

Figure S3. Fully labeled view of the molecular structure of $\mathbf{2}$ at $30 \%$ probability ellipsoids. Hydrogen atoms omitted for clarity. 
Table S5. Crystal data and structure refinement for 2 .

Identification code

Empirical formula

Formula weight

Temperature

Wavelength

Crystal system

Space group

Unit cell dimensions

Volume

Z

Density (calculated)

Absorption coefficient

$\mathrm{F}(000)$

Crystal size

Theta range for data collection

Index ranges

Reflections collected

Independent reflections

Completeness to theta $=39.67^{\circ}$

Absorption correction

Max. and min. transmission

Refinement method

Data / restraints / parameters

Goodness-of-fit on $\mathrm{F}^{2}$

Final $\mathrm{R}$ indices $[\mathrm{I}>2 \operatorname{sigma}(\mathrm{I})]$

$\mathrm{R}$ indices (all data)

Largest diff. peak and hole wb17

C29 H39 Ir N2

607.82

173(2) K

$0.71073 \AA$

Triclinic

P-1
$\mathrm{a}=10.7968(4) \AA$
$\alpha=70.603(2)^{\circ}$.
$\mathrm{b}=11.7022(5) \AA$
$\beta=69.381(2)^{\circ}$.
$\mathrm{c}=12.7810(5) \AA$
$\gamma=66.310(2)^{\circ}$.

$1349.37(9) \AA^{3}$

2

$1.496 \mathrm{Mg} / \mathrm{m}^{3}$

$4.965 \mathrm{~mm}^{-1}$

608

$0.20 \times 0.15 \times 0.10 \mathrm{~mm}^{3}$

1.75 to $39.67^{\circ}$.

$-19<=\mathrm{h}<=19,-20<=\mathrm{k}<=20,-22<=\mathrm{l}<=19$

52539

$15437[\mathrm{R}(\mathrm{int})=0.0231]$

$94.3 \%$

Semi-empirical from equivalents

0.6366 and 0.4367

Full-matrix least-squares on $\mathrm{F}^{2}$

15437 / 0 / 339

0.875

$\mathrm{R} 1=0.0212, \mathrm{wR} 2=0.0513$

$\mathrm{R} 1=0.0298, \mathrm{wR} 2=0.0550$

2.250 and -0.957 e. $\AA^{-3}$ 
Table 3. Bond lengths $[\AA]$ and angles $\left[^{\circ}\right]$ for wb17.

\begin{tabular}{|c|c|c|c|}
\hline $\operatorname{Ir}(1)-\mathrm{N}(1)$ & $2.0431(12)$ & $C(22)-C(23)$ & $1.394(2)$ \\
\hline $\operatorname{Ir}(1)-\mathrm{N}(2)$ & $2.0528(12)$ & $C(23)-C(27)$ & $1.500(2)$ \\
\hline $\operatorname{Ir}(1)-C(28)$ & $2.1260(15)$ & $C(24)-C(26)$ & $1.522(3)$ \\
\hline $\operatorname{Ir}(1)-C(27)$ & $2.1627(15)$ & $C(24)-C(25)$ & $1.530(3)$ \\
\hline $\operatorname{Ir}\left(1^{\prime}\right)-\mathrm{N}(1)$ & $2.191(4)$ & $C(27)-C(28)$ & $1.416(2)$ \\
\hline $\operatorname{Ir}\left(1^{\prime}\right)-C(13)$ & $2.351(5)$ & $C(27)-C(29)$ & $1.505(2)$ \\
\hline $\operatorname{Ir}\left(1^{\prime}\right)-C(12)$ & $2.373(5)$ & & \\
\hline $\operatorname{Ir}\left(1^{\prime}\right)-\mathrm{N}(2)$ & $2.392(4)$ & $\mathrm{N}(1)-\operatorname{Ir}(1)-\mathrm{N}(2)$ & $89.44(5)$ \\
\hline $\mathrm{N}(1)-\mathrm{C}(2)$ & $1.3284(18)$ & $\mathrm{N}(1)-\operatorname{Ir}(1)-\mathrm{C}(28)$ & $158.64(6)$ \\
\hline $\mathrm{N}(1)-\mathrm{C}(6)$ & $1.4451(18)$ & $N(2)-\operatorname{Ir}(1)-C(28)$ & $100.18(6)$ \\
\hline $\mathrm{N}(2)-\mathrm{C}(4)$ & $1.3418(18)$ & N(1)-Ir(1)-C(27) & $162.62(6)$ \\
\hline $\mathrm{N}(2)-\mathrm{C}(18)$ & $1.4208(17)$ & $N(2)-\operatorname{Ir}(1)-C(27)$ & $79.73(5)$ \\
\hline $\mathrm{C}(1)-\mathrm{C}(2)$ & $1.508(2)$ & $\mathrm{C}(28)-\operatorname{Ir}(1)-\mathrm{C}(27)$ & $38.54(6)$ \\
\hline $\mathrm{C}(2)-\mathrm{C}(3)$ & $1.404(2)$ & $\mathrm{N}(1)-\operatorname{Ir}\left(1^{\prime}\right)-\mathrm{C}(13)$ & $106.63(16)$ \\
\hline $\mathrm{C}(3)-\mathrm{C}(4)$ & $1.3869(19)$ & $\mathrm{N}(1)-\operatorname{Ir}\left(1^{\prime}\right)-\mathrm{C}(12)$ & $78.60(13)$ \\
\hline $\mathrm{C}(4)-\mathrm{C}(5)$ & $1.512(2)$ & $C(13)-\operatorname{Ir}\left(1^{\prime}\right)-C(12)$ & $37.60(10)$ \\
\hline $\mathrm{C}(6)-\mathrm{C}(7)$ & $1.402(2)$ & $N(1)-\operatorname{Ir}\left(1^{\prime}\right)-\mathrm{N}(2)$ & $77.81(13)$ \\
\hline$C(6)-C(11)$ & $1.406(2)$ & $\mathrm{C}(13)-\operatorname{Ir}\left(1^{\prime}\right)-\mathrm{N}(2)$ & $171.5(2)$ \\
\hline $\mathrm{C}(7)-\mathrm{C}(8)$ & $1.393(2)$ & $\mathrm{C}(12)-\operatorname{Ir}\left(1^{\prime}\right)-\mathrm{N}(2)$ & $150.47(18)$ \\
\hline$C(7)-C(12)$ & $1.519(2)$ & $\mathrm{C}(2)-\mathrm{N}(1)-\mathrm{C}(6)$ & $117.45(12)$ \\
\hline $\mathrm{C}(8)-\mathrm{C}(9)$ & $1.388(3)$ & $\mathrm{C}(2)-\mathrm{N}(1)-\operatorname{Ir}(1)$ & $125.64(10)$ \\
\hline$C(9)-C(10)$ & $1.380(3)$ & $\mathrm{C}(6)-\mathrm{N}(1)-\operatorname{Ir}(1)$ & $116.90(9)$ \\
\hline $\mathrm{C}(10)-\mathrm{C}(11)$ & $1.396(2)$ & $C(2)-N(1)-\operatorname{Ir}\left(1^{\prime}\right)$ & $103.07(14)$ \\
\hline $\mathrm{C}(11)-\mathrm{C}(15)$ & $1.510(2)$ & $\mathrm{C}(6)-\mathrm{N}(1)-\operatorname{Ir}\left(1^{\prime}\right)$ & $107.49(13)$ \\
\hline $\mathrm{C}(12)-\mathrm{C}(14)$ & $1.518(3)$ & $\operatorname{Ir}(1)-\mathrm{N}(1)-\operatorname{Ir}\left(1^{\prime}\right)$ & $59.13(13)$ \\
\hline $\mathrm{C}(12)-\mathrm{C}(13)$ & $1.523(3)$ & $\mathrm{C}(4)-\mathrm{N}(2)-\mathrm{C}(18)$ & $120.52(12)$ \\
\hline $\mathrm{C}(15)-\mathrm{C}(16)$ & $1.520(3)$ & $\mathrm{C}(4)-\mathrm{N}(2)-\operatorname{Ir}(1)$ & $123.11(9)$ \\
\hline$C(15)-C(17)$ & $1.526(3)$ & $\mathrm{C}(18)-\mathrm{N}(2)-\operatorname{Ir}(1)$ & $113.57(9)$ \\
\hline$C(18)-C(23)$ & $1.397(2)$ & $\mathrm{C}(4)-\mathrm{N}(2)-\operatorname{Ir}\left(1^{\prime}\right)$ & $108.49(14)$ \\
\hline C(18)-C(19) & $1.404(2)$ & $\mathrm{C}(18)-\mathrm{N}(2)-\operatorname{Ir}\left(1^{\prime}\right)$ & $117.59(12)$ \\
\hline C(19)-C(20) & $1.395(2)$ & $\operatorname{Ir}(1)-\mathrm{N}(2)-\operatorname{Ir}\left(1^{\prime}\right)$ & $55.55(11)$ \\
\hline C(19)-C(24) & $1.521(2)$ & $\mathrm{N}(1)-\mathrm{C}(2)-\mathrm{C}(3)$ & $123.48(13)$ \\
\hline$C(20)-C(21)$ & $1.384(3)$ & $\mathrm{N}(1)-\mathrm{C}(2)-\mathrm{C}(1)$ & $120.30(13)$ \\
\hline$C(21)-C(22)$ & $1.390(3)$ & $C(3)-C(2)-C(1)$ & $116.20(13)$ \\
\hline
\end{tabular}




\begin{tabular}{|c|c|c|c|}
\hline $\mathrm{C}(4)-\mathrm{C}(3)-\mathrm{C}(2)$ & $128.36(13)$ & $\mathrm{C}(16)-\mathrm{C}(15)-\mathrm{C}(17)$ & $110.15(16)$ \\
\hline $\mathrm{N}(2)-\mathrm{C}(4)-\mathrm{C}(3)$ & $122.97(12)$ & $\mathrm{C}(23)-\mathrm{C}(18)-\mathrm{C}(19)$ & $120.86(13)$ \\
\hline $\mathrm{N}(2)-\mathrm{C}(4)-\mathrm{C}(5)$ & $120.82(13)$ & $\mathrm{C}(23)-\mathrm{C}(18)-\mathrm{N}(2)$ & $114.42(12)$ \\
\hline$C(3)-C(4)-C(5)$ & $116.07(12)$ & $\mathrm{C}(19)-\mathrm{C}(18)-\mathrm{N}(2)$ & $124.48(13)$ \\
\hline$C(7)-C(6)-C(11)$ & $121.86(13)$ & $\mathrm{C}(20)-\mathrm{C}(19)-\mathrm{C}(18)$ & $116.94(15)$ \\
\hline $\mathrm{C}(7)-\mathrm{C}(6)-\mathrm{N}(1)$ & $118.74(12)$ & $\mathrm{C}(20)-\mathrm{C}(19)-\mathrm{C}(24)$ & $120.52(14)$ \\
\hline $\mathrm{C}(11)-\mathrm{C}(6)-\mathrm{N}(1)$ & $119.37(13)$ & $\mathrm{C}(18)-\mathrm{C}(19)-\mathrm{C}(24)$ & $122.00(13)$ \\
\hline $\mathrm{C}(8)-\mathrm{C}(7)-\mathrm{C}(6)$ & $118.07(14)$ & $\mathrm{C}(21)-\mathrm{C}(20)-\mathrm{C}(19)$ & $121.86(15)$ \\
\hline $\mathrm{C}(8)-\mathrm{C}(7)-\mathrm{C}(12)$ & $120.28(15)$ & $\mathrm{C}(20)-\mathrm{C}(21)-\mathrm{C}(22)$ & $119.92(14)$ \\
\hline $\mathrm{C}(6)-\mathrm{C}(7)-\mathrm{C}(12)$ & $121.63(13)$ & $\mathrm{C}(21)-\mathrm{C}(22)-\mathrm{C}(23)$ & $119.47(16)$ \\
\hline $\mathrm{C}(9)-\mathrm{C}(8)-\mathrm{C}(7)$ & $121.04(17)$ & $\mathrm{C}(22)-\mathrm{C}(23)-\mathrm{C}(18)$ & $119.33(15)$ \\
\hline $\mathrm{C}(10)-\mathrm{C}(9)-\mathrm{C}(8)$ & $119.93(15)$ & $\mathrm{C}(22)-\mathrm{C}(23)-\mathrm{C}(27)$ & $122.68(14)$ \\
\hline $\mathrm{C}(9)-\mathrm{C}(10)-\mathrm{C}(11)$ & $121.41(16)$ & $\mathrm{C}(18)-\mathrm{C}(23)-\mathrm{C}(27)$ & $117.22(12)$ \\
\hline$C(10)-C(11)-C(6)$ & $117.68(15)$ & $C(26)-C(24)-C(19)$ & $108.47(15)$ \\
\hline$C(10)-C(11)-C(15)$ & $120.18(14)$ & $\mathrm{C}(26)-\mathrm{C}(24)-\mathrm{C}(25)$ & $110.14(19)$ \\
\hline C(6)-C(11)-C(15) & $122.14(13)$ & $C(19)-C(24)-C(25)$ & $114.53(15)$ \\
\hline$C(14)-C(12)-C(7)$ & $112.57(15)$ & $\mathrm{C}(28)-\mathrm{C}(27)-\mathrm{C}(23)$ & $113.13(14)$ \\
\hline$C(14)-C(12)-C(13)$ & $111.45(19)$ & $\mathrm{C}(28)-\mathrm{C}(27)-\mathrm{C}(29)$ & $120.93(16)$ \\
\hline$C(7)-C(12)-C(13)$ & $110.53(16)$ & $\mathrm{C}(23)-\mathrm{C}(27)-\mathrm{C}(29)$ & $118.01(15)$ \\
\hline C(14)-C(12)-Ir(1') & $143.74(18)$ & C(28)-C(27)-Ir(1) & $69.33(9)$ \\
\hline$C(7)-C(12)-\operatorname{Ir}\left(1^{\prime}\right)$ & $99.23(14)$ & $C(23)-C(27)-\operatorname{Ir}(1)$ & $108.11(9)$ \\
\hline$C(13)-C(12)-\operatorname{Ir}\left(1^{\prime}\right)$ & $70.40(18)$ & $C(29)-C(27)-\operatorname{Ir}(1)$ & $117.20(12)$ \\
\hline$C(12)-C(13)-\operatorname{Ir}\left(1^{\prime}\right)$ & $71.99(17)$ & $C(27)-C(28)-\operatorname{Ir}(1)$ & $72.13(9)$ \\
\hline$C(11)-C(15)-C(16)$ & $111.92(15)$ & & \\
\hline $\mathrm{C}(11)-\mathrm{C}(15)-\mathrm{C}(17)$ & $111.90(17)$ & & \\
\hline
\end{tabular}




\section{References}

${ }^{1}$ Pangborn, A.; Giardello, M.; Grubbs, R. H.; Rosen, R.; Timmers, F. Organometallics 1996, 15, 1518.

${ }^{2}$ Bernskoetter, W. H.; Lobkovsky, E.; Chirik, P. J. Chem. Comm. 2004, 764.

${ }^{3}$ Feldman, J; McLain, S. J.; Parthasarathy, A.; Marshall, W. J.; Calabrese, J. C.; Arthur; S. D. Organometallics, 1997, 16, 1514.

${ }^{4}$ Carey, D. T.; Cope-Eatough, E. K.; Vilaplana-Maf, E.; Mair, F. S.; Pritchard, R. G.; Warren, J. E.; Woods, R. J. Dalton Trans. 2003, 1083.

${ }^{5}$ Budzelaar, P. H. M.; van Oorta, A. B.; Orpen, A. G. Eur. J. Inorg. Chem. 1998, 1485.

${ }^{6}$ Herde, J. L.; Lambert, J. C.; Senoff, C. V. Inorg. Syn., 1974, 15, 18. 